PROCEEDINGS OF THE

AMERICAN MATHEMATICAL SOCIETY

Volume 128, Number 2, Pages 337-346

S 0002-9939(99)05151-5

Article electronically published on July 6, 1999

\title{
EQUIVALENCES FOR BLOCKS OF THE WEYL GROUPS
}

\author{
RADHA KESSAR
}

(Communicated by Ronald M. Solomon)

\begin{abstract}
We describe the source algebras of the blocks of the Weyl groups of type B and type D in terms of the source algebras of the blocks of the symmetric groups. As a consequence, we show that Puig's conjecture on the finiteness of the number of isomorphism classes of source algebras for blocks of finite groups with a fixed defect group holds for these classes of groups. We also show how certain isomorphisms between subalgebras of block algebras of the symmetric groups can be lifted to block algebras of the Weyl groups of type B.
\end{abstract}

\section{INTRODUCTION}

Let $p$ be a prime number and let $(\mathcal{O}, K, k)$ be a $p$-modular system, i.e. $\mathcal{O}$ is a complete discrete valuation ring with maximal ideal $J(\mathcal{O}), k=\mathcal{O} / J(\mathcal{O})$ is its residue field with characteristic $p$ and $K$ is its field of quotients with characteristic zero; we assume that $k$ is algebraically closed. By a $p$-block of a finite group $G$, we mean a central idempotent of the group algebra $\mathcal{O} G$. Recall that a source algebra of $b$ is an algebra of the form $i \mathcal{O} G i$, where $P$ is a defect group of $G$ and $i$ is a primitive idempotent of $(\mathcal{O} G b)^{P}$, such that $b$ is in the image of the relative trace map $\operatorname{Tr}_{P}^{G}\left((O G)^{P} i(O G)^{P}\right)$. Equivalently, $i$ is characterized as belonging to a local point of $P$ on $\mathcal{O} G b$. A source algebra $i \mathcal{O} G i$ of $b$ is naturally an interior $P$-algebra via the homomorphism which maps an element $x$ of $P$ to the element $i x$ of $i \mathcal{O} G i$ (for details see $[\mathrm{T}], \S 38$ ).

The Weyl group $B_{n}$ is the wreath product of the group of order 2 with $S_{n}$. We will find it convenient to use the following description for it. For a finite set $U$, we denote by $E(U)$ the finite elementary abelian 2-group with a distinguished basis $U$, i.e.

$$
E(U)=\left\langle u: u \in U, u^{2}=1, u v u v=1\right\rangle,
$$

by $E^{0}(U)$, the subgroup of $E(U)$ of index two which is generated by elements $u v$, $u, v \in U$, and by $S(U)$ the symmetric group of the set $U$.

Given $E(U)$, the action of $S(U)$ on the elements of $U$ naturally extends to an action of $S(U)$ on $E(U)$ (via ${ }^{\sigma}(u v)={ }^{\sigma} u^{\sigma} v$ ) and this action leaves invariant the subgroup $E^{0}(U)$. If we put

$$
B(U):=E(U) \ltimes S(U)
$$

Received by the editors March 30, 1998.

1991 Mathematics Subject Classification. Primary 20Cxx.

(C)1999 American Mathematical Society 
and

$$
D(U):=E^{0}(U) \ltimes S(U),
$$

then $B(U)$ and $D(U)$ are respectively the Weyl groups $W\left(B_{|U|}\right)$ and $W\left(D_{|U|+1}\right)$.

For a subset $X$ of $U$, let us denote by $\tau_{X}$ the trivial character of $E(X)$ and by $\tau_{X}^{*}$ the irreducible character of $E(X)$ which takes the value -1 on any element $x$ of $X$. Let $e_{X}$ and $e_{X}^{*}$ denote the central idempotents of $K E(X)$ corresponding respectively to the character $\tau_{X}$ and the character $\tau_{X}^{*}$.

The block theory of $B(U)$ divides naturally into the case where $p=2$ and the case where $p$ is odd. Since $E(U)$ is a normal 2-subgroup of $B(U)$ which is its own centralizer, $B(U)$ has only one 2-block. On the other hand, when $p$ is odd, Clifford theory easily yields a parametrization of the $p$-blocks of $B(U)$ in terms of the $p$-blocks of the symmetric groups (see $[\mathrm{JK}],[\mathrm{P} 1]$ ). We state below the relevant results, which were first described in [O1] and [O2].

From now on, unless we explicitly state otherwise, we assume that $p$ is an odd prime. Let $U=U_{1} \cup U_{2}$ be a partition of $U$ into disjoint nonempty subsets. Since $p$ is odd the central idempotents $e_{U_{1}}$ and $e_{U_{2}}^{*}$ of $K E\left(U_{1}\right)$ and $K E\left(U_{2}\right)$ respectively are blocks of $E\left(U_{1}\right)$ and $E\left(U_{2}\right)$; hence $e_{U_{1}} e_{U_{2}}^{*}$ is a block of $E(U)$ and, further, the stabilizer of $e_{U_{1}} e_{U_{2}}^{*}$ under the conjugation action of $B(U)$ on the algebra $\mathcal{O} E(U)$ is given by

$$
\operatorname{Stab}_{B(U)}\left(e_{U_{1}} e_{U_{2}}^{*}\right)=B\left(U_{1}\right) \times B\left(U_{2}\right) .
$$

For $i=1,2$ let $\tilde{b}_{i}$ be a block of $S\left(U_{i}\right)$. Clearly, since $E(U)$ is abelian, the idempotent $e_{U_{1}} e_{U_{2}}^{*} \tilde{b}_{1} \tilde{b}_{2}$ is a block of $B\left(U_{1}\right) \times B\left(U_{2}\right)$ and it follows by the theory of covering blocks (see Theorem 5.10, Chapter 5, of [NT]) that the idempotent

$$
b:=\operatorname{Tr}_{B\left(U_{1}\right) \times B\left(U_{2}\right)}^{B(U)}\left(e_{U_{1}} e_{U_{2}}^{*} \tilde{b}_{1} \tilde{b}_{2}\right)
$$

is a block of $B(U)$ and that all blocks of $B(U)$ can be described in this way.

It also follows that, if for $i=1,2, P_{i}$ is a defect group of $\tilde{b}_{i}$, then $P:=P_{1} \times P_{2}$ is a defect group of $b$.

Let us fix a partition $U=U_{1} \cup U_{2}$ of $U$ into disjoint subsets, and a block $b$ of $B(U)$ as defined above. We also fix a defect group $P=P_{1} \times P_{2}$ of $b$ as above.

The associate of the block $b$ is the block of $B(U)$ given by

$$
b^{a}:=\operatorname{Tr}_{B\left(U_{1}\right) \times B\left(U_{2}\right)}^{B(U)}\left(e_{U_{1}}^{*} e_{U_{2}} \tilde{b}_{1} \tilde{b}_{2}\right) .
$$

Note that $b$ is equal to its own associate if and only if there is an involution $\sigma$ of $S(U)$ such that ${ }^{\sigma} U_{1}=U_{2}$ and ${ }^{\sigma} \tilde{b}_{1}=\tilde{b}_{2}$. In the following theorem, whenever $b$ is self-associate, we will assume a fixed choice of such a $\sigma$. Also, we will assume in this case that $P_{2}={ }^{\sigma} P_{1}$.

Theorem 1.1. (i) For $i=1,2$ let $t_{i}$ belong to a local point of $P_{i}$ on $\mathcal{O} S\left(U_{i}\right) \tilde{b}_{i}$. Then the interior $P_{1} \times P_{2}$-algebra $t_{1} \mathcal{O} S\left(U_{1}\right) t_{1} \otimes_{\mathcal{O}} t_{2} \mathcal{O} S\left(U_{1}\right) t_{2}$ is isomorphic to a source algebra of the block $b$ of $B(U)$.

(ii) If $b$ is not self-associate, then $b^{\prime}:=b+b^{a}$ is a block of $D(U)$ with defect group $P$, and there is an interior $D(U)$-algebra isomorphism

$$
\mathcal{O} D(U) b^{\prime} \simeq \mathcal{O} B(U) b
$$

which induces an interior P-algebra isomorphism between a source algebra for $b$ and a source algebra of $b^{\prime}$. 
(iii) If $b$ is self-associate, and $P$ is not the identity group, the idempotent $b$ is a block of $\mathcal{O} D(U)$. Further, if $t_{1}$ and $t_{2}$ in (i) are such that $t_{2}={ }^{\sigma} t_{1}$ then a source algebra for $b$ when $b$ is considered as a block of $D(U)$ is isomorphic as interior Palgebra to the crossed product of $t_{1} \mathcal{O} S\left(U_{1}\right) t_{1} \otimes_{\mathcal{O}} t_{2} \mathcal{O} S\left(U_{1}\right) t_{2}$ with the group algebra $\mathcal{O}\langle\sigma\rangle$. In other words, it is isomorphic to the algebra which as $\mathcal{O}$-module is the module

$$
t_{1} \mathcal{O} S\left(U_{1}\right) t_{1} \otimes_{\mathcal{O}} t_{2} \mathcal{O} S\left(U_{1}\right) t_{2} \otimes_{\mathcal{O}} \mathcal{O}\langle\sigma\rangle
$$

with multiplication defined by

$$
\begin{gathered}
\left(a_{1} \otimes a_{2} \otimes \sigma\right)\left(a_{1}^{\prime} \otimes a_{2}^{\prime} \otimes \sigma^{i}\right)=a_{1} \sigma a_{2}^{\prime} \otimes a_{2}{ }^{\sigma} a_{1}^{\prime} \otimes \sigma \sigma^{i}, \\
\left(a_{1} \otimes a_{2} \otimes 1_{\langle\sigma\rangle}\right)\left(a_{1}^{\prime} \otimes a_{2}^{\prime} \sigma^{i}\right)=a_{1} a_{1}^{\prime} \otimes a_{2} a_{2}^{\prime} \otimes \sigma^{i},
\end{gathered}
$$

for $a_{1}, a_{1}^{\prime}$ in $A_{1}, a_{2}, a_{2}^{\prime}$ in $A_{2}, i=1,2$, and where the interior P-algebra structure is given by

$$
g_{1} \times g_{2} \rightarrow g_{1} 1_{t_{1} \mathcal{O} S\left(U_{1}\right) t_{1}} \otimes{ }^{\sigma} g_{2} 1_{t_{2} \mathcal{O} S\left(U_{1}\right) t_{2}} \otimes 1_{\langle\sigma\rangle},
$$

for $g_{1}$ in $P_{1}$ and $g_{2}$ in $P_{2}$.

Corollary 1.2. Puig's conjecture (see [B2]) is true for the blocks of the Weyl groups of type $B$ and type $D$ for all primes $p$. Hence, it holds for all Weyl groups. In other words, for a fixed p-group $P$ there are only finitely many isomorphism classes of interior P-algebras that are source algebras for blocks of the Weyl groups which have defect groups isomorphic to $P$.

In $[\mathrm{S}]$ Scopes showed that the existence of a certain combinatorial relationship between two blocks of two symmetric groups which have isomorphic defect groups forces the corresponding block algebras to be Morita equivalent. In [P2], Puig extended these results to show that this combinatorial relationship in fact forces an explicit isomorphism between certain subalgebras of the block algebras of the concerned blocks. The next theorem is a Clifford theoretic "lift" of Puig's result to the Weyl groups of type $B$. We will say that two blocks of two symmetric groups are Scopes related if they are connected to each other in the same way as the two blocks of Theorem 1.7 of [P2].

Let $b$ be as before a block of $B(U)$ and suppose that $V_{1}$ is a subset of $U_{1}$ such that $\tilde{c}_{1}$ is a block of $S\left(V_{1}\right)$ with $P_{1}$ as defect group. Put $V:=V_{1} \cup U_{2}$ and let $c$ be the block of $B(V)$ defined by

$$
c:=\operatorname{Tr}_{B\left(V_{1}\right) \times B\left(U_{2}\right)}^{B(V)}\left(e_{U_{1}} \tilde{c}_{1} \otimes e_{U_{2}}^{*} \tilde{b}_{2}\right) .
$$

Note that $c \mathcal{O} B(U) b c$ is naturally an interior $B(V) \times B(U-V)$-algebra via the map which sends an element $x$ of $B(V) \times B(U-V)$ to the element $x b c$ of $c \mathcal{O} B(U) b c$, and we shall assume this interior $B(V) \times B(U-V)$-algebra structure for $c \mathcal{O} B(U) b c$ in the theorem below. Also, note that since $S(U-V)$ is a quotient of $B(U-V)$, $\mathcal{O} S(U-V)$ is naturally a $B(U-V)$ module, and this gives $\operatorname{End}_{\mathcal{O}}(\mathcal{O} S(U-V))$ an interior $B(U-V)$ algebra structure.

We are now ready to state our theorem.

Theorem 1.3. Suppose that $\tilde{b}_{1}$ and $\tilde{c}_{1}$ are Scopes related. Then we have an isomorphism of interior $B(V) \times B(U-V)$-algebras

$$
\mathcal{O} B(V) c \otimes_{\mathcal{O}} \operatorname{End}_{\mathcal{O}}(\mathcal{O} S(U-V)) \simeq c \mathcal{O} B(U) b c .
$$




\section{Some RESUlts ON THE INDUCTION OF INTERIOR $G$-ALGEBRAS}

All the results in this section can either be found in section 16 of [T] or are easy consequences of results given there. Hence, except for Proposition 2.4, we omit giving proofs.

Let $G$ be a finite group, $H$ a subgroup of $G$ and $B$ an interior $G$-algebra. Recall that the algebra $\operatorname{Ind}_{H}^{G}(B)$ is an interior $G$-algebra which as an $\mathcal{O}$-module is the $\mathcal{O}$-module $\mathcal{O} G \otimes_{\mathcal{O} H} B \otimes_{\mathcal{O} H} \mathcal{O} G$ with multiplication defined by

$$
(x \otimes t \otimes y)\left(x^{\prime} \otimes t^{\prime} \otimes y^{\prime}\right)= \begin{cases}x \otimes t y x^{\prime} t^{\prime} \otimes y^{\prime} & \text { if } y x^{\prime} \in H, \\ 0 & \text { if } y x^{\prime} \notin H\end{cases}
$$

for $x, x^{\prime}, y, y^{\prime}$ in $G$ and $t$ in $B$; the interior $G$-algebra structure of $\operatorname{Ind}_{H}^{G}(B)$ is defined by the map

$$
\phi: G \rightarrow \operatorname{Ind}_{H}^{G}(B), \quad g \rightarrow \sum_{x \in G / H} g x \otimes 1_{B} \otimes x^{-1} .
$$

The $(\mathcal{O} G, \mathcal{O} G)$ bimodule structure on $\operatorname{Ind}_{H}^{G}(B)$ induced by left and right multiplication by elements $\phi(g)$ coincides with the natural $(\mathcal{O} G, \mathcal{O} G)$ bimodule structure on $\mathcal{O} G \otimes_{\mathcal{O} H} B \otimes_{\mathcal{O} H} \mathcal{O} G$. In other words, we have

$$
\phi(g)\left(f \otimes t \otimes f^{\prime}\right)=g f \otimes t \otimes f^{\prime} \text { and }\left(f \otimes t \otimes f^{\prime}\right) \phi(g)=g f \otimes t \otimes f^{\prime} g .
$$

For the next lemma, recall that if $A$ is an interior $G$-algebra, $X$ a subgroup of $G$ and $j$ an idempotent of $A^{X}$, then $j A j$ is naturally an interior $X$-algebra via the map which sends the element $x$ of $X$ to the element $x j$ of $j A j$.

Lemma 2.1. Let $H$ be a subgroup of $G$ and $B$ an interior $H$-algebra. Put $A=$ $\operatorname{Ind}_{H}^{G}(B)$. Further, suppose that $X$ and $Y$ are subgroups of $G$ with $X \cap H=Y$, and that $i$ is an idempotent of $B^{Y}$. Then a left transversal of $Y$ in $X$ can be extended to a left transversal of $H$ in $G$. In $A$, let $j=\sum_{x \in X / Y} x \otimes i \otimes x^{-1}$. Then $j$ is an idempotent of $A^{X}$, and further, there is a canonical isomorphism of interior $X$-algebras,

$$
\operatorname{Ind}_{Y}^{X}(i B i) \simeq j A j, \quad x \otimes t \otimes y \rightarrow x \otimes t \otimes y \quad(x, y \in X, t \in i B i) .
$$

We also have

Lemma 2.2. Let $D$ be a finite group, $T$ an interior $D$-algebra and $B$ an interior $H$-algebra. There is an isomorphism of interior $G \times D$-algebras

$$
\operatorname{Ind}_{H \times D}^{G \times D}\left(B \otimes_{\mathcal{O}} T\right) \simeq \operatorname{Ind}_{H}^{G}(B) \otimes_{\mathcal{O}} T, \quad x \otimes(t \otimes s) \otimes y \rightarrow(x \otimes t \otimes y) \otimes s
$$

for $x, y$ in $G, t$ in $B$, and $s$ in $T$.

A central point in the proof of Theorem 1.1 will be the following proposition, which is Proposition 16.6 of [T].

Proposition 2.3. Let $A$ be an interior $G$-algebra and let $H$ be a subgroup of $G$. Assume that there exists an idempotent $i$ of $A^{H}$ such that $1_{A}=\operatorname{Tr}_{H}^{G}(i)$ and $i{ }^{g} i=0$ for all $g \in G-H$. Then there is an isomorphism of interior $G$-algebras

$$
\operatorname{Ind}_{H}^{G}(i A i) \simeq A, \quad x \otimes t \otimes y \rightarrow x t y \quad(x, y \in G, t \in i A i) .
$$

The above proposition immediately gives the following Clifford theoretic result. 
Proposition 2.4. Let $E$ be a normal subgroup of $G, e$ a block of $E$ and $H$ the stabilizer of $e$ in $G$. Let $i$ be a block of $H$ covering e and put $b=\operatorname{Tr}_{H}^{G}(i)$. Then there is an isomorphism of interior $G$-algebras

$$
\operatorname{Ind}_{H}^{G}(\mathcal{O} H i) \simeq \mathcal{O} G b, \quad x \otimes t \otimes y \rightarrow x t y \quad(x, y \in G, t \in \mathcal{O} H i) .
$$

Proof. Let $g \in G-H$. Then

$$
i^{g} i=i g i g^{-1}=i e g e i g^{-1}=i e g e g^{-1} \text { gig }^{-1}=0
$$

since $H$ is the stablizer of $e$ in $G$ and $i$ is a block of $H$ covering $e$. Then applying Proposition 2.3 with $A=\mathcal{O} G b$, we get immediately that there is an isomorphism of interior $G$-algebras

$$
\operatorname{Ind}_{H}^{G}(i \mathcal{O} G b i) \simeq \mathcal{O} G b, \quad x \otimes t \otimes y \rightarrow x t y \quad(x, y \in G, t \in i \mathcal{O} G i) .
$$

But the algebra $i \mathcal{O} G b i$ is the algebra $i \mathcal{O} H i$, since for any $g \in G-H$, we have shown above that $i g i=0$. This proves the proposition.

\section{Proof of Theorem 1.1}

We use the notation of Theorem 1.1. It is clear from Proposition 2.4 and from the description of $b$ that there is an isomorphism of interior $\mathcal{O} B(U)$-algebras

$$
\begin{gathered}
\operatorname{Ind}_{B\left(U_{1}\right) \times B\left(U_{2}\right)}^{B(U)} \mathcal{O}\left(B\left(U_{1}\right) \times B\left(U_{2}\right)\right) e_{U_{1}} e_{U_{2}}^{*} \tilde{b}_{1} \tilde{b}_{2} \simeq \mathcal{O} B(U) b, \\
x \otimes t \otimes y \rightarrow x t y \quad\left(x, y \in B(U), t \in \mathcal{O}\left(B\left(U_{1}\right) \times B\left(U_{2}\right)\right) e_{U_{1}} e_{U_{2}}^{*} \tilde{b}_{1} \tilde{b}_{2}\right) .
\end{gathered}
$$

Now, for $i=1,2$, let $t_{i}$ be an element of a local point of $P_{i}$ on $\mathcal{O} S\left(U_{i}\right) \tilde{b}_{i}$. Since

$$
\left(\mathcal{O} S\left(U_{1}\right) \tilde{b}_{1} \otimes_{\mathcal{O}} \mathcal{O} S\left(U_{2}\right) \tilde{b}_{2}\right)^{P} \simeq\left(\mathcal{O} S\left(U_{1}\right) \tilde{b}_{1}\right)^{P_{1}} \otimes_{\mathcal{O}}\left(\mathcal{O} S\left(U_{2}\right) \tilde{b}_{2}\right)^{P_{2}}
$$

and

$$
\begin{aligned}
& \left(\left(\mathcal{O} S\left(U_{1}\right) \tilde{b}_{1}\right)^{P_{1}} \otimes_{\mathcal{O}}\left(\mathcal{O} S\left(U_{2}\right) \tilde{b}_{2}\right)^{P_{2}}\right) / J\left(\left(\mathcal{O} S\left(U_{1}\right) \tilde{b}_{1}\right)^{P_{1}} \otimes_{\mathcal{O}}\left(\mathcal{O} S\left(U_{2}\right) \tilde{b}_{2}\right)^{P_{2}}\right) \\
& \left.\left.\quad \simeq\left(\mathcal{O} S\left(U_{1}\right) \tilde{b}_{1}\right)^{P_{1}}\right) / J\left(\left(\mathcal{O} S\left(U_{1}\right) \tilde{b}_{1}\right)^{P_{1}}\right) \otimes_{k}\left(\mathcal{O} S\left(U_{2}\right) \tilde{b}_{2}\right)^{P_{2}}\right) / J\left(\left(\mathcal{O} S\left(U_{2}\right) \tilde{b}_{2}\right)^{P_{2}}\right),
\end{aligned}
$$

we see that $t_{1} \otimes t_{2}$ is a primitive idempotent of $\left(\mathcal{O} S\left(U_{1}\right) \tilde{b}_{1} \otimes_{\mathcal{O}} \mathcal{O} S\left(U_{2}\right) \tilde{b}_{2}\right)^{P}$. Similarly, we have an isomorphism of Brauer quotients

$$
\operatorname{Br}_{P}\left(\left(\mathcal{O} S\left(U_{1}\right) \tilde{b}_{1} \otimes_{\mathcal{O}} \mathcal{O} S\left(U_{2}\right) \tilde{b}_{2}\right)^{P}\right) \simeq \operatorname{Br}_{P_{1}}\left(\left(\mathcal{O} S\left(U_{1}\right) \tilde{b}_{1}\right)^{P_{1}}\right) \otimes_{k} \operatorname{Br}_{P_{2}}\left(\left(\mathcal{O} S\left(U_{2}\right) \tilde{b}_{2}\right)^{P_{2}}\right) .
$$

In other words, $t_{1} \otimes t_{2}$ belongs to a local point of $P$ on $\mathcal{O} S\left(U_{1}\right) \tilde{b}_{1} \otimes_{\mathcal{O}} \mathcal{O} S\left(U_{2}\right) \tilde{b}_{2}$; hence, using the canonical isomorphism

$$
\mathcal{O} S\left(U_{1}\right) \tilde{b}_{1} \otimes_{\mathcal{O}} \mathcal{O} S\left(U_{2}\right) \tilde{b}_{2} \simeq \mathcal{O}\left(S\left(U_{1}\right) \times S\left(U_{2}\right)\right) \tilde{b}_{1} \tilde{b}_{2}, \quad a_{1} \otimes a_{2} \rightarrow a_{1} a_{2},
$$

we have that $t_{1} t_{2}$ belongs to a local point of $P$ on $\mathcal{O}\left(S\left(U_{1}\right) \times S\left(U_{2}\right)\right) \tilde{b}_{1} \tilde{b}_{2}$. Further, multiplying the term on the left of the above isomorphism on both sides with $t_{1} \otimes t_{2}$ and the term on the right on both sides with $t_{1} t_{2}$ yields an isomorphism of interior $P$-algebras

$$
t_{1} \mathcal{O} S\left(U_{1}\right) t_{1} \otimes_{\mathcal{O}} t_{2} \mathcal{O} S\left(U_{2}\right) t_{2} \simeq t_{1} t_{2} \mathcal{O}\left(S\left(U_{1}\right) \times S\left(U_{2}\right)\right) t_{1} t_{2} .
$$

On the other hand, notice that if we consider $\mathcal{O}\left(B\left(U_{1}\right) \times B\left(U_{2}\right)\right) e_{U_{1}} e_{U_{2}}^{*} \tilde{b}_{1} \tilde{b}_{2}$ as an interior $S\left(U_{1}\right) \times S\left(U_{2}\right)$-algebra, then $\mathcal{O}\left(B\left(U_{1}\right) \times B\left(U_{2}\right)\right) e_{U_{1}} e_{U_{2}}^{*} \tilde{b}_{1} \tilde{b}_{2}$ is isomorphic to the algebra $\mathcal{O}\left(S\left(U_{1}\right) \times S\left(U_{2}\right)\right) \tilde{b}_{1} \tilde{b}_{2}$ since for any $u$ in $E(U), u e_{U_{1}} e_{U_{2}}^{*}= \pm e_{U_{1}} e_{U_{2}}^{*}$; in particular these are isomorphic as interior $P$-algebras. Hence, $t_{1} t_{2}$ belongs to a 
local point of $P$ on $\mathcal{O}\left(B\left(U_{1}\right) \times B\left(U_{2}\right)\right) e_{U_{1}} e_{U_{2}}^{*} \tilde{b}_{1} \tilde{b}_{2}$ and we have an isomorphism of interior $P$-algebras

$$
t_{1} t_{2} \mathcal{O}\left(S\left(U_{1}\right) \times S\left(U_{2}\right)\right) t_{1} t_{2} \simeq t_{1} t_{2} \mathcal{O}\left(B\left(U_{1}\right) \times B\left(U_{2}\right)\right) t_{1} t_{2}
$$

Further, by parts (d) and (f) of Proposition 15.1 of [T], $1 \otimes t_{1} t_{2} \otimes 1$ belongs to a local point of $P$ on $\operatorname{Ind}_{B\left(U_{1}\right) \times B\left(U_{2}\right)}^{B(U)} \mathcal{O}\left(B\left(U_{1}\right) \times B\left(U_{2}\right)\right) e_{U_{1}} e_{U_{2}}^{*} \tilde{b}_{1} \tilde{b}_{2}$. Since there is clearly an interior $P$-algebra isomorphism

$$
\begin{gathered}
1 \otimes t_{1} t_{2} \otimes 1\left(\operatorname{Ind}_{B\left(U_{1}\right) \times B\left(U_{2}\right)}^{B(U)} \mathcal{O}\left(B\left(U_{1}\right) \times B\left(U_{2}\right)\right)\right) 1 \otimes t_{1} t_{2} \otimes 1 \\
\simeq t_{1} t_{2} \mathcal{O}\left(B\left(U_{1}\right) \times B\left(U_{2}\right)\right) t_{1} t_{2},
\end{gathered}
$$

by multiplying on both sides the term on the left of (3.1) with $1 \otimes t_{1} t_{2} \otimes 1$ and the term on the right of (3.1) with $t_{1} t_{2}$, and using the isomorphisms (3.2) and (3.3) we get part (i) of Theorem 1.1.

We put $e:=e_{U_{1}} e_{U_{2}}^{*}+e_{U_{1}}^{*} e_{U_{2}}$. Then it is clear that $e$ is a block of $E^{0}(U)$. For a finite group $G$, and a block $b$ of $G$, recall that $\operatorname{Irr}(G, b)$ denotes the set of ordinary irreducible characters of $G$ that belong to the block $b$.

By the definition of $b$ it is clear that every element $\chi$ of $\operatorname{Irr}(B(U), b)$ is the induced character

$$
\operatorname{Ind}_{B\left(U_{1}\right) \times B\left(U_{2}\right)}^{B(U)}\left(\tau_{U_{1}} \tau_{U_{2}}^{*} \chi_{1} \chi_{2}\right)
$$

for some elements $\chi_{1}$ of $\operatorname{Irr}\left(S\left(U_{1}\right), \tilde{b}_{1}\right)$ and $\chi_{2}$ of $\operatorname{Irr}\left(S\left(U_{2}\right), \tilde{b}_{2}\right)$. For the character $\chi$ as above let

$$
\chi^{a}:=\operatorname{Ind}_{B\left(U_{1}\right) \times B\left(U_{2}\right)}^{B(U)}\left(\tau_{U_{1}}^{*} \tau_{U_{2}} \chi_{1} \chi_{2}\right) .
$$

By Mackey decomposition the restriction of $\chi$ to $D(U)$ is given by

$$
\begin{aligned}
\left.\operatorname{Res}\right|_{D(U)}(\chi) & =\operatorname{Ind}_{B\left(U_{1}\right) \times B\left(U_{2}\right) \cap D(U)}^{D(U)}\left(\tau_{U_{1}} \tau_{U_{2}}^{*} \chi_{1} \chi_{2}\right) \\
& =\operatorname{Ind}_{E^{0}(U) \ltimes\left(S\left(U_{1}\right) \times S\left(U_{2}\right)\right)}^{D(U)} \tau_{U_{1}} \tau_{U_{2}}^{*}\left(\chi_{1} \chi_{2}\right) \\
& =\operatorname{Ind}_{E^{0}(U) \ltimes\left(S\left(U_{1}\right) \times S\left(U_{2}\right)\right)}^{D(U)} \tau_{U_{1}}^{*} \tau_{U_{2}}\left(\chi_{1} \chi_{2}\right) \\
& =\operatorname{Ind}_{B(U)}^{D(U) \times B\left(U_{2}\right) \cap D(U)}\left(\tau_{U_{1}}^{*} \tau_{U_{2}} \chi_{1} \chi_{2}\right) \\
& =\operatorname{Res}_{D(U)}\left(\chi^{a}\right) .
\end{aligned}
$$

Also, a character $\chi$ of $B(U)$ belongs to the block $b$ of $B(U)$ if and only if $\chi^{a}$ belongs to the block $b^{a}$. So, if $b \neq b^{a}$, then in particular $\chi \neq \chi^{a}$ for any $\chi$ in $\operatorname{Irr}(B(U), b)$ and using Clifford theory it follows by (3.4) that $\operatorname{Res}_{D(U)}(\chi)=$ $\operatorname{Res}_{D(U)}\left(\chi^{a}\right)$ is irreducible for all $\chi$ in $\operatorname{Irr}(B(U), b)$. In particular, $b$ and $b^{a}$ each cover exactly one block of $D(U)$.

On the other hand, it is clear that

$$
b^{\prime}:=b+b^{a}=\operatorname{Tr}_{S\left(U_{1}\right) \times S\left(U_{2}\right)}^{S(U)} e \tilde{b}_{1} \tilde{b}_{2}
$$

is a central idempotent of $D(U)$. This proves that $b^{\prime}$ is a block of $D(U)$; it is clear that $P$ is a defect group of $b^{\prime}$.

It also follows from the above analysis that

$$
\operatorname{Irr}\left(D(U), b^{\prime}\right)=\left\{\left.\operatorname{Res}\right|_{D(U)} \chi: \chi \in \operatorname{Irr}(B(U), b)\right\} .
$$

In other words, restriction provides a one to one correspondence between $\operatorname{Irr}(B(U), b)$ and $\operatorname{Irr}\left(D(U), b^{\prime}\right)$. So, by Theorem 0.1 of [B1], it follows that the block algebras 
$\mathcal{O} D(U) b^{\prime}$ and $\mathcal{O} B(U) b$ are isomorphic via the map which sends an element $a$ of $\mathcal{O} D(U) b^{\prime}$ to the element $a b$ of $\mathcal{O} B(U) b$. This is clearly an isomorphism of interior $D(U)$-algebras and in particular, it induces an interior $P$-algebra isomorphism between a source algebra of $b^{\prime}$ and a source algebra of $b$. This proves part (ii) of Theorem 1.1.

The proof of the third part of Theorem 1.1 depends heavily on the following result which is a special case of Theorem 5.5 of [P2].

Proposition 3.1. Let $L$ be a normal subgroup of $N$ and let $f$ be a block of $L$ normalized by $N$. Assume that $C_{N}(P) \subset L$ where $P$ is a defect group of $f$. Let $\gamma$ be a local point of $P$ on $\mathcal{O} L f$ and $H_{\beta}$ a pointed group on $\mathcal{O} L f$ such that $P_{\gamma} \subset H_{\beta}$. Then $\beta$ is contained in a point of $H$ on $\mathcal{O} N$, i.e. elements of $\beta$ remain primitive in the algebra $(\mathcal{O} N)^{H}$.

Proof. See [P2], Theorem 5.5 (i) and Remark 5.6.

Note that if we take $H=L$, the above theorem yields that if $C_{N}(P) \subset L$, then $f$ is a block of $N$.

Let us assume from now on that $b$ is self-associate and let $\sigma$ be as in part (iii) of Theorem 1.1. Clearly, ${ }^{\sigma}\left(e_{U_{1}} e_{U_{2}}^{*}\right)=e_{U_{1}}^{*} e_{U_{2}}$; hence if we denote by $T$ the group $\left(S\left(U_{1}\right) \times S\left(U_{2}\right)\right) \ltimes\langle\sigma\rangle$, then it is clear from the description of $b$ that

$$
b=\operatorname{Tr}_{E^{0}(U) \ltimes T}^{D(U)}\left(e \tilde{b}_{1} \tilde{b}_{2}\right) .
$$

Now the idempotent $e \tilde{b}_{1} \tilde{b}_{2}$ is a block of the normal subgroup $E^{0}(U) \ltimes\left\langle S\left(U_{1}\right) \times\right.$ $\left.S\left(U_{2}\right)\right\rangle$ of $E^{0}(U) \ltimes T$, and it has $P$ as a defect group. Since we are under the assumption that $P=P_{1} \times P_{2}$ is not the identity group, we certainly have

$$
C_{E^{0}(U) \ltimes T}(P)=C_{E^{0}(U) \ltimes\left\langle S\left(U_{1}\right) \times S\left(U_{2}\right)\right\rangle}(P)
$$

and hence by Proposition 3.1 applied with $L=E^{0}(U) \ltimes\left\langle S\left(U_{1}\right) \times S\left(U_{2}\right)\right\rangle, N=$ $E^{0}(U) \ltimes T, f=e \tilde{b}_{1} \tilde{b}_{2}$ and $H=L$, we have that $e \tilde{b}_{1} \tilde{b}_{2}$ is a block of $E^{0}(U) \ltimes T$. Since $E^{0}(U) \ltimes T$ is the stabilizer in $D(U)$ of the block $e$ of $E^{0}(U)$, it follows by Theorem 5.5, Chapter 5 of [NT] and by (3.5), that $b$ is a block of $D(U)$.

So, applying Proposition 2.4 with $E=E^{0}(U), H=E^{0} \ltimes T$, and $i=e \tilde{b}_{1} \tilde{b}_{2}$, we get that there is an isomorphism of interior $D(U)$-algebras

$$
\operatorname{Ind}_{E^{0}(U) \ltimes T}^{D(U)} e \tilde{b}_{1} \tilde{b}_{2} \mathcal{O}\left(E^{0}(U) \ltimes T\right) \simeq \mathcal{O} D(U) b .
$$

In particular, it follows just as for (i) that a source algebra for $b$ as a block of $D(U)$ is isomorphic as interior $P$-algebra to a source-algebra for the block $e \tilde{b}_{1} \tilde{b}_{2}$ of $E^{0}(U) \ltimes T$, and this in turn is clearly isomorphic to a source algebra for the block $\tilde{b}_{1} \tilde{b}_{2}$ of the group $T$.

On the other hand, let $t_{1}$ and $t_{2}={ }^{\sigma} t_{1}$ be as in Theorem 1.1 (iii). Then, just as for the proof of (i), $t_{1} t_{2}$ belongs to a local point of $P$ on $S\left(U_{1}\right) \times S\left(U_{2}\right)$. Now, in view of (3.6), it follows by Proposition 3.1 applied with $L=S\left(U_{1}\right) \times S\left(U_{2}\right)$, $f=\tilde{b}_{1} \tilde{b}_{2}, N=T$ and $H=P$ that $t_{1} t_{2}$ belongs to a local point of $P$ on $\mathcal{O} T \tilde{b}_{1} \tilde{b}_{2}$. In other words, $t_{1} t_{2} \mathcal{O} T t_{1} t_{2}$ is a source algebra for the block $\tilde{b}_{1} \tilde{b}_{2}$ of the group $T$.

Define the $\mathcal{O}$-linear map

$$
\phi: t_{1} \mathcal{O} S\left(U_{1}\right) t_{1} \otimes_{\mathcal{O}} t_{2} \mathcal{O} S\left(U_{2}\right) t_{2} \otimes_{\mathcal{O}} \mathcal{O}\langle\sigma\rangle \rightarrow t_{1} t_{2} \mathcal{O} T t_{1} t_{2}, \quad a \otimes a^{\prime} \otimes \sigma^{i} \rightarrow a a^{\prime} \sigma^{i}
$$


for $a \in t_{1} \mathcal{O} S\left(U_{1}\right) t_{1}, a^{\prime} \in t_{2} \mathcal{O} S\left(U_{2}\right) t_{2}$ and $i=1,2$. Then, since $t_{2}={ }^{\sigma} t_{1}$, it is easy to check that $\phi$ is an isomorphism of interior $P$-algebras. This completes the proof of part (iii) of Theorem 1.1.

It remains to prove Corollary 1.2. Since the groups $B(U)$ and $D(U)$ each have only one 2-block we only need to prove the result when $p$ is odd. Let us fix a nonidentity $p$-group $P$ and let $b$ be a block of $B(U)$. Then, by Theorem 1.1 there are $p$-groups $P_{1}$ and $P_{2}$ such that $P=P_{1} \times P_{2}$ and a source algebra for $b$ is isomorphic to the tensor product $A_{1} \otimes A_{2}$ of the source algebras $A_{1}$ and $A_{2}$ of blocks of two symmetric groups which have $P_{1}$ and $P_{2}$ respectively as defect groups. By paragraph 1.8 of [P2], there are only finitely many possibilities (up to interior $P_{i^{-}}$ algebra isomorphism) for each $A_{i}$, and since, for a fixed $P$, there are only finitely many possibilities for $P_{1}$ and $P_{2}$ the result holds for the $p$-blocks of the groups $B(U)$. The result for the groups $D(U)$ follows in the same way since by parts (ii) and (iii) the source algebras of a block of $D(U)$ are isomorphic either to a tensor product of source algebras of blocks of symmetry of these source algebras with the group algebra of the group of order 2. Since the only infinite families of Weyl groups are the symmetric groups and the groups of type $B$ and $D$, it follows that Puig's conjecture is true for the Weyl groups.

\section{Proof of Theorem 1.3}

By the description of $b$ and $c$, we get that in $\mathcal{O} B(U)$,

$$
\begin{aligned}
& b c=b \operatorname{Tr}_{B\left(V_{1}\right) \times B\left(U_{2}\right)}^{B(V)} e_{V_{1}} e_{U_{2}}^{*} \tilde{c}_{1} \tilde{b}_{2} \\
& =\operatorname{Tr}_{B\left(V_{1}\right) \times B\left(U_{2}\right)}^{B(V)} b e_{V_{1}} e_{U_{2}}^{*} \tilde{c}_{1} \tilde{b}_{2} \\
& =\operatorname{Tr}_{B\left(V_{1}\right) \times B\left(U_{2}\right)}^{B(V)}\left(\operatorname{Tr}_{B\left(U_{1}\right) \times U_{2}}^{B(U)}\left(e_{U_{1}} e_{U_{2}}^{*}\right) \tilde{b}_{1} \tilde{b}_{2}\right) e_{V_{1}} e_{U_{2}}^{*} \tilde{c}_{1} \tilde{b}_{2} \\
& =\operatorname{Tr}_{B\left(V_{1}\right) \times B\left(U_{2}\right)}^{B(V)}\left(\sum_{x \in S(U) / S\left(U_{1}\right) \times S\left(U_{2}\right)}\left(e{ }^{x} U_{1} e^{*}{ }^{*} U_{2}\right){ }^{x} \tilde{b}_{1}{ }^{x} \tilde{b}_{2}\right) e_{V_{1}} e_{U_{2}}^{*} \tilde{c}_{1} \tilde{b}_{2} \\
& =\operatorname{Tr}_{B\left(V_{1}\right) \times B\left(U_{2}\right)}^{B(V)}\left(\sum_{x \in S(U) / S\left(U_{1}\right) \times S\left(U_{2}\right)}{ }^{x} \tilde{b}_{1}{ }^{x} \tilde{b}_{2} e^{x}{ }_{U_{1}} e^{*}{ }_{{ }^{\prime}}{ }_{U_{2}} e_{V_{1}} e_{U_{2}}^{*} \tilde{c}_{1} \tilde{b}_{2}\right) .
\end{aligned}
$$

Now for any $x$ in $S(U), e{ }^{x} U_{1} e^{*}{ }_{x}{ }_{U_{2}} e_{V_{1}} e_{U_{2}}^{*}=e^{x} U_{U_{1}} e^{*}{ }_{{ }}{ }_{2}$ if $V_{1} \subset{ }^{x} U_{1}$ and $U_{2} \subset{ }^{x} U_{2}$ and is zero otherwise. Moroever, $U_{2} \subset{ }^{x} U_{2}$ if and only if $x \in S\left(U_{1}\right) \times S\left(U_{2}\right)$. Hence, from (4.1) we get that

$$
b c=\operatorname{Tr}_{B\left(V_{1}\right) \times B\left(U_{2}\right)}^{B(V)}\left(e_{U_{1}} e_{U_{2}}^{*} \tilde{c}_{1} \tilde{b}_{1} \tilde{b}_{2}\right) .
$$

It is clear from (4.2) that in $\mathcal{O} B(U) b$, the idempotent $b c$ is the image under the isomorphism in (3.1) of the element

$$
j:=\sum_{x \in B(V) / B\left(V_{1}\right) \times B\left(U_{2}\right)} x \otimes e_{U_{1}} e_{U_{2}}^{*} \tilde{b}_{1} \tilde{c}_{1} \tilde{b}_{2} \otimes x^{-1}
$$

of $\operatorname{Ind}_{B\left(U_{1}\right) \times B\left(U_{2}\right)}^{B(U)} \mathcal{O}\left(B\left(U_{1}\right) \times B\left(U_{2}\right)\right) e_{U_{1}} e_{U_{2}}^{*} \tilde{b}_{1} \tilde{b}_{2}$ and that $j$ is centralized by the group $B(V) \times B(U-V)$. Hence, multiplying the algebra on the left of (3.1) on both sides by the idempotent $j$ and the algebra on the right of (3.1) on both sides by the idempotent $b c$, we get an isomorphism of interior $B(V) \times B(U-V)$-algebras

$$
j\left(\operatorname{Ind}_{B\left(U_{1}\right) \times B\left(U_{2}\right)}^{B(U)} \mathcal{O}\left(B\left(U_{1}\right) \times B\left(U_{2}\right)\right) e_{U_{1}} e_{U_{2}}^{*} \tilde{b}_{1} \tilde{c}_{1} \tilde{b}_{2}\right) j \simeq c \mathcal{O} B(U) b c .
$$


On the other hand, by Lemma 2.1 applied with $G=B(U), H=B\left(U_{1}\right) \times$ $B\left(U_{2}\right), B=\mathcal{O}\left(B\left(U_{1}\right) \times B\left(U_{2}\right)\right) e_{U_{1}} e_{U_{2}}^{*} \tilde{b}_{1} \tilde{b}_{2}, X=B(V) \times B(U-V), Y=B\left(V_{1}\right) \times$ $B(U-V) \times B\left(U_{2}\right)$ and $i=e_{U_{1}} e_{U_{2}}^{*} \tilde{b}_{1} \tilde{c}_{1} \tilde{b}_{2}$, we get that there is an isomorphism of interior $B(V) \times B(U-V)$-algebras

$$
\begin{aligned}
& j\left(\operatorname{Ind}_{B\left(U_{1}\right) \times B\left(U_{2}\right)}^{B(U)} \mathcal{O}\left(B\left(U_{1}\right) \times B\left(U_{2}\right)\right) e_{U_{1}} e_{U_{2}}^{*} \tilde{b}_{1} \tilde{b}_{2}\right) j \\
& \quad \simeq \operatorname{Ind}_{B\left(V_{1}\right) \times B(U-V) \times(U-V) \times B\left(U_{2}\right)}^{B(V)} \tilde{c}_{1} \mathcal{O}\left(B\left(U_{1}\right) \times B\left(U_{2}\right)\right) e_{U_{1}} e_{U_{2}}^{*} \tilde{b}_{1} \tilde{c}_{1} \tilde{b}_{2}
\end{aligned}
$$

and hence, composing the inverse of this isomorphism with the isomorphism of (4.3), we get an isomorphism of interior $B(V) \times B(U-V)$-algebras

$$
\operatorname{Ind}_{B\left(V_{1}\right) \times B(U-V) \times B\left(U_{2}\right)}^{B(V) \times B(U-V)} \tilde{c}_{1} \mathcal{O}\left(B\left(U_{1}\right) \times B\left(U_{2}\right)\right) e_{U_{1}} e_{U_{2}}^{*} \tilde{b}_{1} \tilde{c}_{1} \tilde{b}_{2} \simeq c \mathcal{O} B(U) b c .
$$

Also, note that there is a canonical interior $B\left(V_{1}\right) \times B(U-V) \times B\left(U_{2}\right)$-algebra isomorphism

$$
\tilde{c}_{1} \mathcal{O}\left(B\left(U_{1}\right) \times B\left(U_{2}\right)\right) e_{U_{1}} e_{U_{2}}^{*} \tilde{b}_{1} \tilde{c}_{1} \tilde{b}_{2} \simeq \tilde{c}_{1} \mathcal{O} B\left(U_{1}\right) e_{U_{1}} \tilde{b}_{1} \tilde{c}_{1} \otimes_{\mathcal{O}} \mathcal{O} B\left(U_{2}\right) e_{U_{2}}^{*} \tilde{b}_{2} .
$$

Now, since $\tilde{b}_{1}$ and $\tilde{c}_{1}$ are Scopes related, by Theorem 1.7 of [P2], we have that there is an interior $S\left(V_{1}\right) \times S(U-V)$-algebra isomorphism

$$
\mathcal{O} S\left(V_{1}\right) \tilde{c}_{1} \otimes_{\mathcal{O}} \operatorname{End}_{\mathcal{O}}(\mathcal{O} S(U-V)) \simeq \tilde{c}_{1} \mathcal{O} S\left(U_{1}\right) \tilde{b}_{1} \tilde{c}_{1} .
$$

For any $x$ in $E\left(V_{1}\right) \times E(U-V), x e_{U_{1}}=e_{U_{1}}$ and $x e_{V_{1}}=e_{V_{1}}$; hence the isomorphism (4.7) yields an interior $B\left(V_{1}\right) \times B(U-V)$-algebra isomorphism

$$
\mathcal{O} B\left(V_{1}\right) e_{V_{1}} \tilde{c}_{1} \otimes_{\mathcal{O}} \operatorname{End}_{\mathcal{O}}(\mathcal{O} S(U-V)) \simeq \tilde{c}_{1} \mathcal{O} B\left(U_{1}\right) e_{U_{1}} \tilde{b}_{1} \tilde{c}_{1} .
$$

Then (4.5), (4.6) and (4.8) yield an interior $B(V) \times B(U-V)$-algebra isomorphism

$$
\begin{aligned}
& \operatorname{Ind}_{B\left(V_{1}\right) \times B(U-V) \times B\left(U_{2}\right)}^{B(V B(U-V)}\left(\mathcal{O} B\left(V_{1}\right) e_{U_{1}} \tilde{c}_{1} \otimes_{\mathcal{O}} \operatorname{End}_{\mathcal{O}}(\mathcal{O} S(U-V)) \otimes_{\mathcal{O}} \mathcal{O} B\left(U_{2}\right) e_{U_{2}}^{*} \tilde{b}_{2}\right) \\
& \quad \simeq c \mathcal{O} B(U) b c .
\end{aligned}
$$

Now, applying Lemma 2.2 with $T=\operatorname{End}_{\mathcal{O}}(\mathcal{O} S(U-V)), H=B\left(V_{1}\right) \times B(U-V)$ $\times B\left(U_{2}\right), G=B(V) \times B(U-V)$, and $B=\mathcal{O} B\left(V_{1}\right) e_{U_{1}} \tilde{c}_{1} \otimes_{\mathcal{O}} \mathcal{O} B\left(U_{2}\right) e_{U_{2}}^{*} \tilde{b}_{2},(4.9)$ yields interior $B(V) \times B(U-V)$-algebra isomorphisms

$$
\begin{aligned}
& c \mathcal{O} B(U) b c \\
& \simeq\left(\operatorname{Ind}_{B\left(V_{1}\right) \times B\left(U_{2}\right)}^{B(V)} \mathcal{O} B\left(V_{1}\right) e_{V_{1}} \tilde{c}_{1} \otimes_{\mathcal{O}} \mathcal{O} B\left(U_{2}\right) e_{U_{2}}^{*} \tilde{b}_{2}\right) \otimes_{\mathcal{O}} \operatorname{End}_{\mathcal{O}}(\mathcal{O} S(U-V)) \\
& \simeq\left(\operatorname{Ind}_{B\left(V_{1}\right) \times B\left(U_{2}\right)}^{B(V)} \mathcal{O}\left(B\left(V_{1}\right) \times B\left(U_{2}\right)\right) e_{V_{1}} e_{U_{2}}^{*} \tilde{c}_{1} \tilde{b}_{2}\right) \otimes_{\mathcal{O}} \operatorname{End}_{\mathcal{O}}(\mathcal{O} S(U-V)) .
\end{aligned}
$$

On the other hand, as for (3.1), we have that

$$
\operatorname{Ind}_{B\left(V_{1}\right) \times B\left(U_{2}\right)}^{B(V)} \mathcal{O}\left(B\left(V_{1}\right) \times B\left(U_{2}\right)\right) e_{V_{1}} e_{U_{2}}^{*} \tilde{c}_{1} \tilde{b}_{2} \simeq \mathcal{O} B(V) c
$$

as interior $B(V)$-algebras.

Then the isomorphisms (4.10) and (4.11) immediately yield the isomorphism desired in (1.1). 


\section{REFERENCES}

[B1] M. Broué, Isométries de caracteres et équivalences de Morita ou dérivées, Publ. Math. I.H.E.S. 71 (1990), 45-63. MR 91i:20007

[B2] Lquivalences of blocks of group algebras, Publ. LMENS-93-4, Paris (1993). MR 97c:20004

[JK] G.D. James and A. Kerber, The representation theory of the symmetric group. Enc. of Math. 16, Addison \& Wesley, London, 1981. MR 83k:20003

[NT] H. Nagao and Y. Tsushima, Representations of finite groups, Academic Press, London, 1989. MR 90h:20008

[O1] M. Osima, On the representations of the generalized symmetric group, Math. J. Okayama Univ. 4 (1955-56), 39-56. MR 16:794d

[O2] - On the representations of the generalized symmetric group II, Math. J. Okayama Univ. 6 (1956-57), 81-97. MR 18:716e

[P1] L. Puig, The Nakayama conjecture and the Brauer pairs, Séminaire sur les Groupes Finis, Pub. Math. Univ. Paris VII, 25, 1987. MR 88i:20018

[P2] - On Joanna Scopes' criterion of equivalence for blocks of symmetric groups, Algebra Colloq. 1:1 (1994), 25-55. MR 95f:20020

[S] J. Scopes, Cartan matrices and Morita equivalence for blocks of the symmetric groups, J. Algebra 142 (1991), 441-455. MR 92h:20023

[T] J. Thévenaz, G-Algebras and Modular Representation Theory, Oxford University Press, Oxford, 1995. MR 96j:20017

Department of Mathematics, University of Minnesota, 206 Church Street, S.E., MinNEAPOLIS, Minnesota 55455

E-mail address: kessar@math.umn.edu 